\title{
PROTOCOLS PERFORMANCE IN NEXT GENERATION NETWORKS
}

Next Generation Network (NGN) is a packet-based network integrating legacy networks and providing "anywhere, anytime and on any device" ubiquitous services. One of the prerequisites of NGN is to support generalized mobility making Mobility Management (MM) a crucial factor towards a seamless provision of multimedia applications. A very promising scenario is the design and implementation of a UMTS / WLAN interworking architecture. In literature a variety of MM protocols operating from different layers of the protocol stack have been proposed. Nevertheless, the comparison of them has been less extensive mainly narrowed between extensions of the same protocol or different basic protocols. In this article a comprehensive comparison between the most efficient approaches of well-known mobility protocols in UMTS/WLAN networks is presented. It is worth noting that the comparison includes not only theoretical but also experimental handoff analysis using OPNET Modeler 15.0 and evaluating the protocols on both non real-time and real-time applications.

\section{Introduction}

A NGN is a packet-based network able to provide services including Telecommunication Services and able to make use of multiple broadband, QoS-enabled transport technologies in which service-related functions are independent from underlying transport-related technologies. It supports generalized mobility which will allow consistent and ubiquitous provision of services to users.

Evolution to NGN is a process in which parts of the existing networks are replaced or upgraded to the corresponding NGN components providing similar or better functionality, while maintaining the services provided by the original network. One of the most attractive ideas is the design and implementation of a UMTS/ WLAN interworking architecture.

In telecommunications, Internet and Mobile Telecommunications services are deemed as the most important breakthroughs over the past decades. Through Third Generation (3G) systems Mobile Telecommunications gained access to the Internet. Universal Mobile Telecommunication System (UMTS) is the most widely deployed $3 \mathrm{G}$ network. UMTS provides high mobility and wide coverage area but lacks in means of data rate and cost. Conversely, Wireless Local Area Networks (WLAN) services are inexpensive and have a high bandwidth but cover small areas and allow limited mobility. These complementary characteristics of both wireless technologies in conjunction with their high penetration prompt the idea of interoperability.

This requires inter-technology MM, which can take place in several layers of communication. MM is divided into two tasks:
Location Management and Handoff Management. The former enables the system to track the locations of Mobile Nodes (MNs) between consecutive communications. The latter is the process by which a MN keeps its connection active when it moves from one access point to another. The most important characteristic of the Handoff Management is the Handoff Delay, which is the time interval between last packet received before the link loss and the first packet received through the new link. In NGN there are two types of handoff: Horizontal and Vertical. A horizontal handoff is a handoff between two network access points (AP) that use the same network technology where as a Vertical handoff is a handoff between APs of different network technologies.

Several MM protocols have been proposed through the years implemented in different layers of protocol stack. The Mobile Internet Protocol (MIP) [1], [2] is the most well known Networklayer solution for MM. Despite the fact that is a mature protocol it has been slowly deployed in real networks as it suffers from high Handoff Delay due to Triangular Routing, high packet loss rate and conflicting security issues. On the other hand, mobile Stream Control Transmission Protocol (mSCTP) [3] is a Transport-layer efficient solution with main characteristic the multi-homing, which allows a MN to be reachable through different IP addresses. Finally, in the Application Layer, the Session Initiation Protocol (SIP) [4] has been proposed as a MM protocol.

The rest of the article is organized as follows. In Section 2 a brief description of the most important candidate protocols for MM is given. In Section 3 a theoretical analysis of Handoff Delay for the most efficient extensions of the basic protocols is presented. In Section 4, the results were obtained through simulation using

\footnotetext{
* Asimakis Lykourgiotis, Stavros Kotsopoulos

Wireless Telecommunications Laboratory, Department of Electrical and Computer Engineering, University of Patras, Greece,

E-mail: asly@ece.upatras.gr
} 
OPNET Modeler 15.0 are discussed. In Section 5, we conclude the paper by summarizing our findings and suggesting potential future research efforts.

\section{Basic Mobility Protocols}

\subsection{Mobile IP}

The MIPv4[1] was introduced to allow the MN to gain access to the Internet by utilizing two IP Addresses, one for identification called Home Address (HoA), the other for routing called Care of Address (CoA). According to MIPv4, every domain must have a router called Home Agent (HA), so as to allow roaming of its users and a router called Foreign Agent (FA), so as to accept visitors. Every time a MN enters a Foreign Network, it registers with the FA and obtains a CoA. Then the FA informs the HA of the MN's current CoA. Every time a Correspondent Node $(\mathrm{CN})$ establishes a connection with the MN, it sends packets to MN through the HA. This deficient way of communication is called Triangular Routing and is one of the main downsides of the protocol. Some other drawbacks of the protocol are the limited number of IP addresses and the vulnerability against malicious attacks. The Mobile IPv6 (MIPv6) [2] was introduced as a standard that surpasses the drawbacks of the previous version. A fundamental sub-protocol of Mobile IPv6 is the Return Routability Protocol (RRP). The RRP is a procedure by which a $\mathrm{CN}$ carries out a minimal verification that a MN owns an address $(\mathrm{HoA})$ and is reachable at another (CoA). With the RRP the $\mathrm{CN}$ can be informed for the CoA and send packets directly to the MN, solving the problem of Triangular Routing. Moreover, MIPv6 also eliminates the use of FA as the $\mathrm{MN}$ is capable of performing its own mobility procedures, involves more sophisticated security procedures and provides a wider range of IP addresses.

\subsection{Mobile SCTP}

Stream Control Transmission Protocol (SCTP) is a Transportlayer protocol which was designed to face the drawbacks of TCP. A dominant characteristic of SCTP is multi-homing, making it a candidate protocol for Handoff Management in all-IP networks. To accomplish that, a new module was added called ADD-IP which leaded to the extension named mSCTP [3]. On the other hand, $\mathrm{mSCTP}$ does not support Location Management and must be used along with another mobility protocol such as MIP or SIP. A connection between two SCTP-endpoints is called an association. Multihoming allows each point of the association to maintain more than one IP Address. Among that set of addresses one is used as Primary Address. The ADD-IP module allows the endpoints to add, delete or change the Primary IP address dynamically during the handoff procedure. As a result, a MN can prepare a new path before the breakdown of the active one and perform a soft handoff. $\mathrm{mSCTP}$ can be used to provide seamless handoff without requiring any additional mobility agents. So, the only requirement for providing the soft handoff is that both the $\mathrm{MN}$ and $\mathrm{CN}$ are equipped with the mSCTP implementations.

\subsection{Session Initiation Protocol}

SIP [4] is a signaling protocol which was initially used to handle multimedia sessions such as Voice over IP (VoIP). SIP was accepted as a $3 \mathrm{GPP}$ signaling protocol and permanent element of the IP Multimedia Subsystem (IMS) architecture for IP based streaming multimedia services [5]. Additionally, SIP resides at the Application Layer and can support MM. SIP introduces several new entities like User Agent (UA), proxy server and registrar. An $\mathrm{UA}$ is an interface agent responsible for initiating, exchanging messages and terminating a SIP session. A proxy server is an intermediate entity that receives a request and forwards it on behalf of the requestor. Proxy server uses location services to trace an UA and checks its ability to communicate. Finally, the registrar server enables clients to alter the address at which they can be contacted. The SIP user address is called Uniform Resource Identifier (URI) and has an email format (user@host). The SIP protocol supports two kinds of handoffs. The former is called Pre-Call as it determines that the MN enters a new foreign network before the initiation of a session. The latter is called Mid-Call because the MN enters a new foreign network while having an ongoing session. In this case the SIP re-INVITE method is used. One of the advantages of SIP is that it supports session mobility without any modifications of lower layer protocols. As for data delivery, with SIP data are exchanged directly between $\mathrm{MN}$ and $\mathrm{CN}$ without involving any tunneling.

\section{Handoff Delay Analysis}

Below, the Handoff Delay analysis of the four more efficient extensions, as indicated in publications, of the forementioned basic protocols is presented. As mentioned before, two types of handoff occur in an integrated UMTS / WLAN architecture: horizontal and vertical. The horizontal handoff procedure can be divided in four stages. Firstly, the MN detects it has moved to a different network (Move Detection - MD) and authenticates itself to an Authentication, Authorization, and Accounting (AAA) server so as to get access to the network. Afterwards, the MN obtains a new IP address (Address Configuration - AC). Finally, MN registers its new location and address (Registration-REG).

In the vertical handoff scheme, assuming that the $\mathrm{MN}$ can use the two different interfaces simultaneously, the MD and AC procedures are performed while the connection with the $\mathrm{CN}$ is continued. Thus, the vertical handoff delay is decreased to the AAA and REG delay. In further analysis, the delay of procedure $X$ is denoted by $T_{X}$ and the one-way transmission delay between node $X$ and $Y$ by $T_{X-Y}$.

\subsection{Route Optimization in MIPv4}

The most important extension of MPIv4 is the Route Optimization in MIPv4 (MIPv4-RO) [6], which solves the Triangular Routing problem. The handoff procedure comprises the following steps. Firstly, the MN moves into a new subnetwork and the MD 
process is initiated. Then the MN performs the AAA process. The MD process is performed by receiving an Agent Advertisement (AA) message from the new Foreign Agent (FA), containing the network prefix. Then, the MN obtains a new Care-of-Address (CoA) from the FA or a DHCP server. In the end, the REG process takes place by sending a Registration Request (RR) to the FA. FA forwards a BU to the HA. The HA replies with a Binding Acknowledgement (BA) message. The FA notifies the MN of the successful registration with a Registration Reply (RRp) message. Direct communication between $\mathrm{MN}$ and $\mathrm{CN}$ is possible through Route Optimization. The $\mathrm{MN}$ can send a registration packet to the $\mathrm{CN}$ to inform it for its new CoA. The $\mathrm{CN}$, then sends a BA to both MN and HA. For our analysis we assume that $T_{H A-C N}<T_{C N-M N}$, and so we use the latter. Therefore, the $T_{R E G}$ is:

$$
T_{R E G}^{M I P V 4-R O}=T_{V H O}=T_{M N-H A}+T_{H A-C N}+T_{C N-M N},
$$

While, the horizontal handoff delay:

$$
D_{H O}^{M I P \vee 4-R O}=T_{M D}+T_{A A A}+T_{A C 1}+T_{R E G}^{M I P V 4-R O},
$$

\subsection{Proxy Mobile IPv6}

PMIPv6 is a protocol implementing MM procedures in network part without involving the MN. For that purpose, two new functional entities are introduced in PMIPv6, the Local Mobility Anchor (LMA) and the Mobility Access Gateway (MAG).The LMA is the topological anchor point for the MN's home network prefix, receiving any packets that are sent to the $\mathrm{MN}$ by any node in or outside the PMIPv6 domain. The LMA is Home Agent with enhanced capabilities for supporting PMIPv6. The MAG is a new functional entity that emulates the MN's home link on the access link. To do so, the MAG sends Router Advertisement messages, containing the MN's home network prefix. Typically, it is a function runs on an Access Router (AR). PMIPv6 supports multihoming by allowing the $\mathrm{MN}$ to connect to a domain through multiple interfaces for simultaneous access. By this means, PMIPv6 does not require move detection and address configuration procedures to be performed during handoff reducing handoff delay and signaling. Additionally, network-based mobility management is compatible with current devices and reduces signaling exchange through the wireless link, so as to accomplish the initial goals.

In this analysis, we investigate only the handoffs occurred between PMIPv6 subnets. When a MN enters the serving area of a MAG performs the AAA. Then the MAG obtains the MN's profile and sends PBU to the LMA. Finally the LMA replies with a Proxy Binding Acknowledgement (PBA) and sets up a bidirectional tunnel with the MAG in order to use the MN's home network prefix over it.

So the registration delay, which is also the vertical handoff delay (VHO), is constrained to the messages exchange between MAG and LMA and the transmission of packets from the MAG to the MN:

$$
T_{R E G}^{P M I P V 6}=T_{V H O}=2 \times T_{M A G-L M A}+T_{M A G-M N},
$$

And the horizontal handoff delay:

$$
D_{H O}^{P M I P v 6}=T_{A A A}+T_{R E G}^{P M I P v 6}
$$

\subsection{Cellular SCTP}

The Cellular SCTP (cSCTP) [9] is an extension of mSCTP. In contrast with $\mathrm{mSCTP}$, the cSCTP does not await the ADDIP_set_primary chunk to be sent to the $\mathrm{CN}$ but sends duplicate packets through old and new path by the receiving of the ADD-IP ASCONF-ACK chunk. With this extension the registration delay is reduced and less packet retransmissions occur as the data are transferred via two different paths reducing the probability of MN's missing the packets. cSCTP is proposed to be used with SIP because, as mSCTP, doesn't support Location Management. Hence, the registration delay for the CSCTP is the time between sending an ASCONF_add_ip chunk and receiving an ASCONF-ACK_add_ip chunk:

$$
T_{R E G}^{c S C T P}=T_{V H O}=2 \times T_{M N-C N},
$$

The horizontal handoff delay is:

$$
D_{H O}^{C S C T P}=T_{M D}+T_{A A A}+T_{A C 2}+T_{R E G}^{c S C T P}
$$

\subsection{Mobility Management Using SIP Extension}

MMUSE [10] is an important extension of SIP as shown by the results. MMUSE introduces a new intermediate entity named Mobility Management Server (MMS) to be in charge of managing terminal mobility. The MMS can be considered as an extended Session Border Controller (SBC). A SBC is a router which is located at the border of the Home Network and acts as a SIP proxy for signaling. With the legacy SIP re-INVITE method signaling messages are exchanged between the MN and the $\mathrm{CN}$, whereas with the MMUSE approach the exchange is between MN and MMS. By controlling the location of MMS, MMUSE decreases the handoff delay. Additionally, as the $\mathrm{CN}$ is not involved in the handoff procedure legacy devices can be used. In MMUSE, when the MN enters a new subnet, it obtains a new IP address through a DHCP server. Afterwards, the registration process is performed where the MN sends a SIP HO-Reg to the MMS, which responds with a 200 OK message. Then, the registration delay is:

$$
T_{R E G}^{M M U S E}=T_{V H O}=2 \times T_{M N-M M S},
$$

And the horizontal handoff delay is:

$$
D_{H O}^{M M U S E}=T_{M D}+T_{A A A}+T_{A C 2}+T_{R E G}^{M M U S E},
$$

For analytical purpose, we will take the following assumption:

1. Based on the protocol architectures the entities introduced from each protocol follow the mapping shown in Fig. 1. For example, the FA location of MIPv4 is the same as the MAG 
of PMIPv6 because both reside in the AR of the Foreign Network.

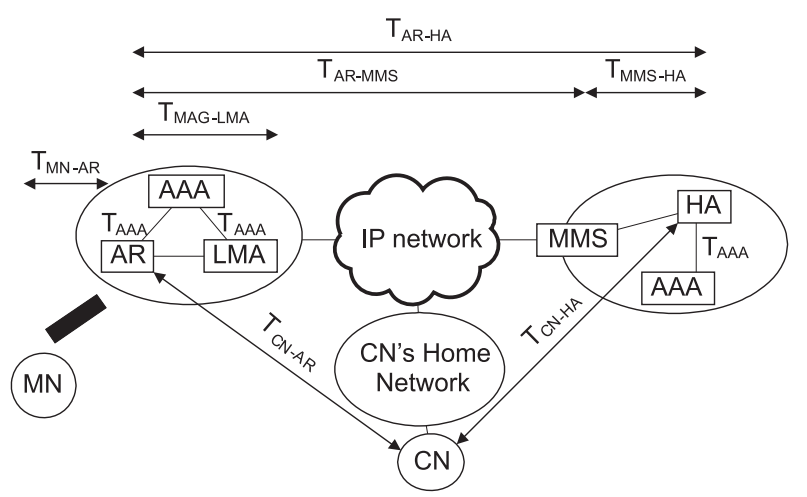

Fig. 1 Architectural Model

2. The delay of DAD procedure is extremely high and it is inefficient to use it. As our main goal is to examine the handoff signaling delay of the protocols we will not take in to account the DAD delay.

3. The AAA procedure (like DIAMETER) is the same for all evaluated protocols.

Moreover, we have made the following calculations:

1. The SIP and mSCTP protocols do not support any MD mechanism and thus they use the one implemented by the Network Layer called Router Discovery. In [2] it is specified that routers must send unsolicited RA in small intervals with MinRtrAdvIntrvl as smaller value and MaxRtrAdvIntrvl as maximum. So the MD mechanism is the same for all the protocols with a mean value of :

$$
T_{M D}=\frac{\text { MaxRirAdvIntrvl }+ \text { MinRtrAdvIntrvl }}{2},
$$

2. For the current analysis, we will consider Stateful Address Configuration through a DHCP server. For MIP-based protocols, this is a one round-trip process, so:

$$
T_{A C 1}=2 \times T_{M N-D H C P},
$$

For SIP-based protocols, this is a two round-trip process, so:

$$
T_{A C 2}=4 \times T_{M N-D H C P},
$$

Fig. 2 summarizes the message flows of the forementioned protocols.

\section{Experimental Handoff Analysis}

The simulation results were obtained by OPNET Modeler 15.0. The main idea was to create a custom node model (named Mobility Node) which intervenes between the MN and $\mathrm{CN}$ and simulates the mobility's protocol behavior similar with [11]. In

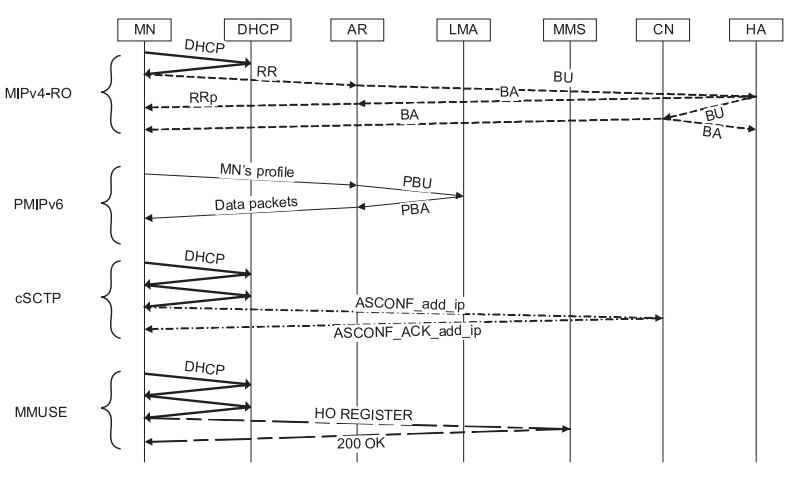

Fig. 2 Message Flow

[11] the assumption that $T_{R E G}$ and $T_{M N-C N}$ are proportional is made. According to previous analysis $T_{R E G}$ is also affected by $T_{M N-H A}$. In our proposed model $T_{R E G}$ is calculated based on our analysis showing the influence of both factors.

In Fig. 3 the Logic Diagram of our proposed model is presented. Each time a new packet arrives to the Mobility Node, it is checked if the packet is a handoff initiation message. If so, the Mobility Node is set to handoff mode. Then, the handoff delay is calculated. The Mobility Node stays in handoff mode for a time interval equals to the handoff delay, dropping every packet that arrives during this interval. The Mobility Node returns to normal mode when the handoff delay time passes. If the arriving packet is a data packet, it is checked if the Mobility Node is in handoff mode. If so, the packet is dropped, if not it is forwarded, adding the related transmission delay. In our simulation, both IEEE 802.11g WLAN and UMTS operate at full mobility $(80 \mathrm{~km} \mid \mathrm{hr})$. That results to a data rate of $384 \mathrm{Kbps}$ for UMTS and $15 \mathrm{Mbps}$ for WLAN. An extra delay is added or subtracted due to wireless link's data rate.

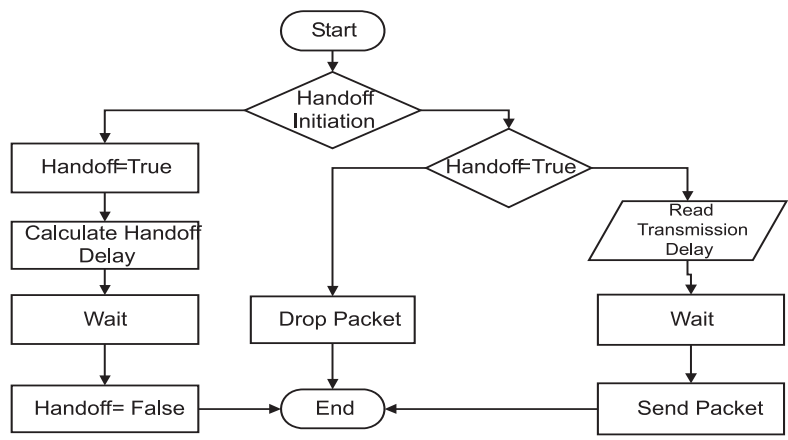

Fig. 3 Logic Diagram

For our analysis, we used the parameters that are outlined in Table 1. All these values are similar to those given in related work in [8], [12].

Firstly, in order to evaluate the four handoff mechanisms we plot the handoff delay as function of $T_{M N-C N}$ (Fig. 4) and as function of $T_{M N-H A}$ (Fig. 5). 
Parameter setting values

\begin{tabular}{|llll|}
\hline Parameter & Value & Parameter & Value \\
\hline$T_{A A A}$ & $\mathbf{1 2} \mathbf{~ m s}$ & $T_{H A-M N}$ & $\mathbf{2 0 ~} \mathbf{~ s}$ \\
$T_{M N-C N}$ & $\mathbf{2 0 ~} \mathbf{~ s}$ & $T_{M N-M M S}$ & $\mathbf{1 0 ~} \mathbf{~ s}$ \\
$T_{M N-D H C P}$ & $\mathbf{1 2} \mathbf{~ m s}$ & $T_{M D}$ & $\mathbf{5 0 ~} \mathbf{~ s}$ \\
$T_{M A G-L M A}$ & $\mathbf{1 0 ~} \mathbf{m s}$ & $T_{H A-C N}$ & $\mathbf{1 0 ~} \mathbf{~ s}$ \\
\hline
\end{tabular}

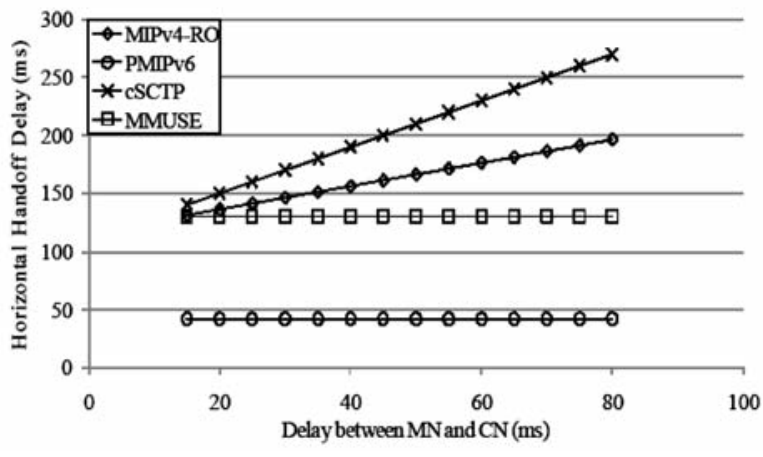

Fig. 4 Handoff Delay vs. delay between $M N$ and $C N$

It can be observed that handoff delay of PMIPv6 is the smallest, because the MD and $\mathrm{AC}$ procedures are not required. Moreover, the protocol is not affected by the change of $T_{M N-C N}$ or $T_{M N-H A}$. On the other hand MIPv4-RO is affected by both factors. The MMUSE is only affected by $T_{M N-H A}$ whereas the CSCTP is only affected by $T_{M N-C N}$. Both protocols have a greater slope than MIPv4-RO.

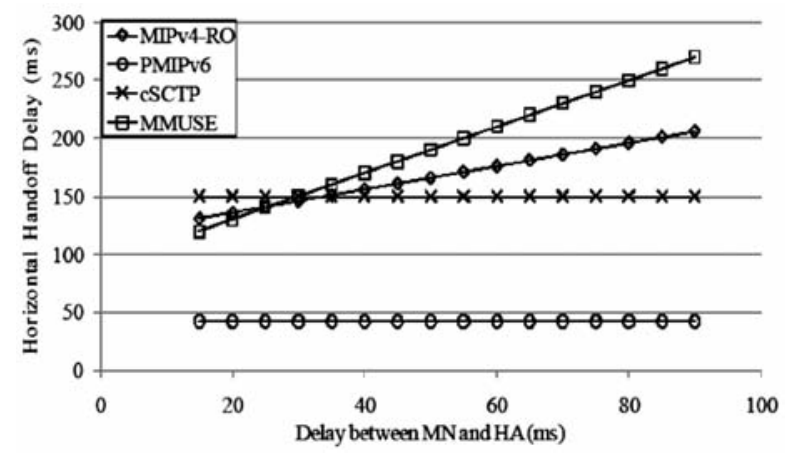

Fig. 5 Handoff Delay vs. delay between $M N$ and $H A$

Consequently, we evaluated the protocols on both non realtime (FTP) and real-time (VoIP) applications. For FTP, the important metric is the throughput which is affected by the transmission delay. For MIPv4-RO, cSCTP and MMUSE the transmission delay is the same as these protocols enabled direct communication between $\mathrm{MN}$ and $\mathrm{CN}$, resulting to same throughput. On the other hand, in PMIPv6 the traffic is sent through the tunnel between
LMA and MAG, resulting to larger transmission delay. Fig. 7 shows the impact of all types of handoff that can occur in a UMTS / WLAN network on throughput. It is observed that PMIPv6 has smaller throughput than other protocols especially in WLAN where the wireless link delay is smaller.

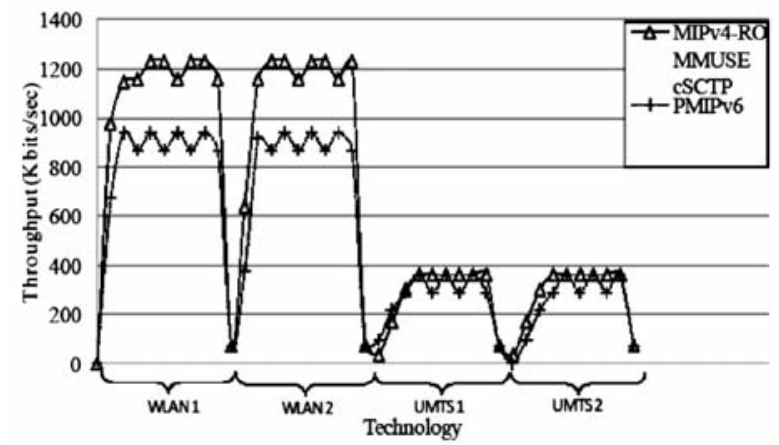

Fig. 7 Throughput vs. Technology

For VoIP we used the G.711 codec which provides a data rate equal to 100 packets/sec and we set $T_{M N-C N}=T_{M N-H A}=30 \mathrm{~ms}$. The important metric in this simulation is the number of lost packets during handoff. As shown in Fig. 8 PMIPv6 achieves less packet loss for all types of handoff.

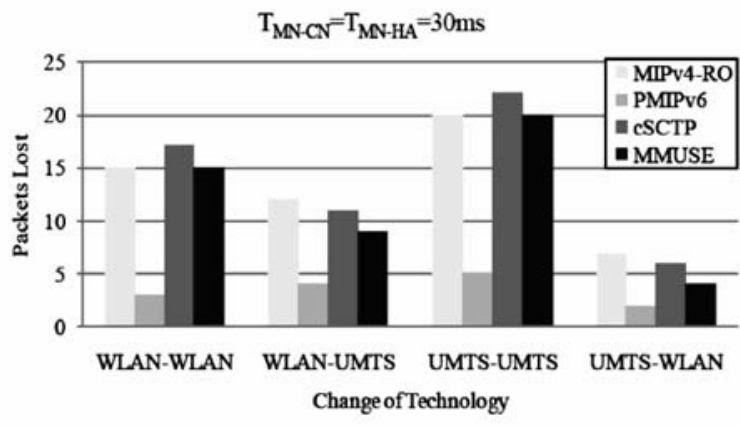

Fig. 8 Lost Packet vs. Type of Handoff

$\left(T_{M N-C N}=T_{M N-H A}=30 \mathrm{~ms}\right.$. $)$

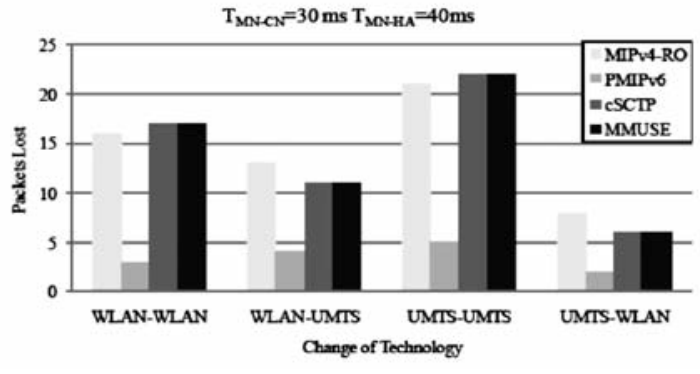

Fig. 9 Lost Packet vs. Type of Handoff

$\left(T_{M N-C N}=30 \mathrm{~ms}, T_{M N-H A}=40 \mathrm{~ms}\right.$. 
Furthermore, we repeated the simulation in order to observe the impact of $T_{M N-H A}$, by increasing its value to $40 \mathrm{~ms}$. As shown in Fig. 9 PMIPv6 and cSCTP are not influenced as Handoff Delay is the same. On the contrary, in MIPv4-RO lost packets increased by one and in MMUSE by two.

Finally, we set $T_{M N-C N}$ to $40 \mathrm{~ms}$. As shown in Fig. 10, in this case PMIPv6 and MMUSE are not influenced as Handoff Delay is the same. On the contrary, in MIPv4-RO lost packets increased by one and in cSCTP by two.

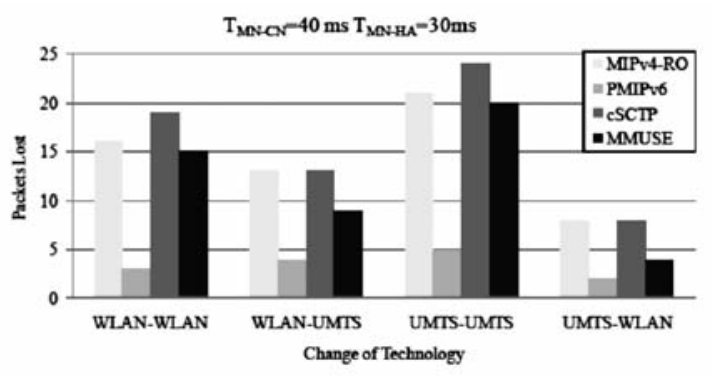

Fig. 10 Lost Packet vs. Type of Handoff

$\left(T_{M N-C N}=40 \mathrm{~ms}, T_{M N-H A}=30 \mathrm{~ms}.\right)$

\section{Conclusions}

To the best of our knowledge, this is the first article that evaluates PMIPv6 against the most efficient protocols for mobility management in integrated UMTS/WLAN networks. In this article, we presented a PMIPv6 protocol overview. Firstly we obtained analytical results for performance evaluation of the candidate protocols and then proceeded to simulations for both real-time and non-real-time applications PMIPv6 is proven to be the best solution, in terms of handoff delay as it does not have any dependence on the distance between MN and both $\mathrm{CN}$ and HA because the $\mathrm{MD}$ and $\mathrm{AC}$ are not required. As a result the PMIPv6 is the protocol with the least packet loss during handoff. Simulation results showed that PMIPv6 is a feasible solution even for real time applications such as VoIP.

However, the deficiency of the protocol is the tunneling between LMA and MAG, adding delay to packet transmission. As a result PMIPv6 provides lower throughput than the other candidate protocols. Simulation results showed that this reduction is greater in WLAN as the wireless link delay is smaller. Moreover, PMIPv6 does not support global mobility and future research should focus on better protocol design to cope with these drawbacks.

\section{References}

[1] PERKINS, C.: IP Mobility Support for IPv4. IETF RFC 3444, 2002.

[2] JOHNSON, D., PERKINS, C., ARKKO, J.: Mobility Support in IPv6, IETF RFC 3775, 2004.

[3] RIEGEL, M., TUEXEN, M.: Mobile SCTP. draft-riegel-tuexen-mobile-sctp-09, 2007.

[4] HANDLEY, M., SCHUlZRINNE, H., SCHOOLER, E., ROSENBERG, J. SIP: Session Initiation Protocol, IETF RFC, 2543. 1999.

[5] KOVACIKOVA, T., SEGEC, P., BRUNCKO, M.: Standardization Paths for NGN IMS-based architecture, Communications - Scientific Letters of the University of Zilina, No. 4, 2008.

[6] PERKINS, C., JOHNSON, D.: Route Optimization in Mobile IP, draft-ietf-mobileip-optim-11.txt, 2001.

[7] GUNDAVELLI, S., LEUNG, ED. K., DEVARAPALLI, V., CHOWDHURY, K., PATIL, B.: Proxy Mobile IPv6, IETF RFC 5213, 2008.

[8] KONG, K., LEE, W., HAN, Y., SHIN, M. (2008). Mobility Management for all-IP Mobile Networks: Mobile IPv6 vs. Proxy mobile IPv6, IEEE Wireless Communications vol. 15, pp. 36-45.

[9] AYDIN, I., SEOK, W., SHEN, C.-C.: Cellular SCTP: A Transport-layer Approach to Internet Mobility, IEEE Int'l Conference on Computer Communications and Networks, pp. 285-290, 2003.

[10] SALSANO, S., POLIDORO, A., MINGARDI, C., NICCOLINI, S., VELTRI, L.: SIP-based Mobility Management in Next Generation Networks, IEEE Wireless Communications, Vol 15, pp. 92-99, 2008.

[11] ABDEL-GHAFFAR, H.S. EBRAHIM, H.A.-A. KHALIFA, A.A.: Efficient Mobility Management Protocols for Integrated UMTS and Outdoor WLAN 802.11g, Wireless Communications and Mobile Computing Conference, pp. 552-558, 2008.

[12] POLIDORO, A., SALSANO, S., NICCOLINI. S.: Performance Evaluation of Vertical Handover Mechanisms in IP Networks, IEEE Wireless Communications and Networking Conference, pp. 2783-2788, 2008. 